\title{
El desarrollo regional en el estado fronterizo de Sonora, México*
}

\author{
Jesús Arroyo Alejandre** \\ Joaquín Bracamontes Nevárez ${ }^{* * *}$
}

\begin{abstract}
Resumen. Este trabajo analiza las desigualdades espaciales en el desarrollo socioeconómico regional de Sonora. Para este propósito, se diseña un modelo estadístico y se recurre a la técnica de análisis factorial de componentes principales, lo que permite estimar un Índice de Desarrollo Socioeconómico Municipal (IDSEM) para el año 2000, lo cual hace evidente la concentración geográfica del desarrollo regional en Sonora. También se especificó un modelo econométrico que permitió identificar los factores que obstaculizan o incentivan el desarrollo socioeconómico de la población en los municipios del Estado.

Palabras clave: población, concentración geográfica, estructura industrial, urbanización, Índice de Desarrollo Socioeconómico Municipal.
\end{abstract}

\begin{abstract}
This work analyzes the spatial inequalities in the regional socioeconomic development of Sonora. In order to do so, a statistical model is designed and it resorts to the technique of the factorial analysis of main components which allows to estimate a Municipal Socioeconomic Development Index (IDSEM) for year 2000, which makes evident the geographic concentration of the regional development in Sonora. An econometric model was specified as well, that allowed to identify the factors that prevent or stimulate the socioeconomic development of the population in the municipalities of the State.

Keywords: population, geographic concentration, industrial structure, urbanization, Municipal Socioeconomic Development Index.
\end{abstract}

\footnotetext{
* Este trabajo comprende una parte de los resultados obtenidos en la tesis de maestría del segundo autor con la dirección del primero. Se agradece al Consejo Nacional de Ciencia y Tecnología (Conacyt), institución que financió los estudios de maestría en Economía en el Centro Universitario de Ciencias EconómicoAdministrativas (CUCEA) de la Universidad de Guadalajara.

** Profesor-investigador en el Departamento de Estudios Regionales (DER) del Instituto de Estudios Económicos y Regionales (Ineser). Actualmente es rector en el Centro Universitario de Ciencias Económico-Administrativas (CUCEA) de la Universidad de Guadalajara y miembro del Sistema Nacional de Investigadores, Nivel III. Correo electrónico: jesusarr@cucea.udg.mx *** Técnico titular en el Departamento de Economía del Centro de Investigación en Alimentación y Desarrollo, A.C. (CIAD-AC) de Hermosillo, Sonora. Correo electrónico: joaco@cascabel.ciad.mx
} 


\section{Introducción}

En el contexto de la globalización económica, la apertura de las economías nacionales ha repercutido de diversas formas en la dinámica económica y social de los diferentes países. En la escala subnacional, dicha apertura se refleja en un impacto diferenciado en los niveles de desarrollo, por lo cual las disparidades regionales siguen siendo motivo de preocupación en el ámbito académico e institucional.

El objetivo principal de este trabajo es analizar las desigualdades espaciales en el desarrollo socioeconómico de Sonora para el año 2000, así como conocer la estructura económica regional. Un objetivo específico es conocer los factores que obstaculizan o incentivan el desarrollo socioeconómico municipal. Otro objetivo específico es identificar aquellos municipios que observan un mayor desarrollo socioeconómico y los que tienen mayor rezago en el Estado.

Las disparidades regionales obedecen a procesos y razones diversas, pero la localización de las actividades económicas puede explicar en mucho las diferencias en el desarrollo. Por tanto, la hipótesis de trabajo argumenta que el desarrollo socioeconómico municipal se debe a la concentración de la industria en los centros urbanos, lo que induce a la concentración geográfica del desarrollo en los municipios más urbanizados y a la vez de la población en los municipios con mayor desarrollo.

El trabajo se divide en cinco partes, incluida esta parte introductoria. La segunda aborda las principales teorías sobre concentración económica y desarrollo regional, así como la revisión de trabajos empíricos en el tema. La tercera parte comprende la explicación metodológica, en la cuarta se analiza el desarrollo socioeconómico municipal en la entidad y los factores que determinan tales niveles de desarrollo. Por último, se exponen las conclusiones del trabajo.

\section{Las teorías y el trabajo empírico sobre concentración económica y desarrollo regional}

En los años posteriores a 1950, la teoría que subyace en el desarrollo regional es la teoría de los polos de crecimiento. Francois Perroux se 
preocupó por explicar cómo las actividades económicas se organizaban por sí mismas en el espacio geográfico. ${ }^{1}$ Apuntaba que el establecimiento de cada empresa era "disperso geográficamente" y que "entre ellas se formaban nexos de organización de fuerza variada"; por ello, argumentaba que "como un campo de fuerzas, el espacio económico consiste de centros o polos de los cuales emanan fuerzas centrífugas y se atraen fuerzas centrípetas" (Perroux, 1950, citado en Miyoshi, 1997: 5).

En esta perspectiva, Perroux afirmó que el desarrollo era desequilibrado y que toma lugar primero en nodos, para luego propagarse a otros objetos. En palabras de Perroux: "El crecimiento no aparece en todas partes al mismo tiempo, aparece en nodos o polos de crecimiento de variada intensidad, éste se propaga por varias vías y con efectos diferentes sobre toda la economía" (Perroux, 1955, citado en Parr, 1999a: 1197 y en Miyoshi, 1997: 5).

El polo de crecimiento se describe como un complejo industrial vinculado productivamente y dominado por una industria propulsora -industrie motrice- que termina siendo el motor del desarrollo por su capacidad para innovar y estimular, y para dominar otras industrias -industries mues- (Parr, 1999a y Meyer, et al., 1996). De tal modo, el polo de crecimiento se plantea en términos de un espacio económico abstracto y la polarización ${ }^{2}$ se mide respecto a la intensidad de las transacciones interfirmas o interindustrias.

No obstante, Perroux reconocía la posibilidad de que un polo de crecimiento en el espacio económico pudiera también existir como una entidad territorial con posibles implicaciones, esto es, como una ciudad o región (Parr, 1999a). En este sentido, Perroux no concibe el polo de crecimiento como un fenómeno aislado y se enfoca sobre las relaciones de estas unidades propulsoras -industrie motrice-, señalando que

${ }^{1}$ Perroux ofrece una triple tipología del espacio: a) espacio como área de planeación de la unidad de decisión; $b$ ) espacio como campo de fuerzas actuante sobre las unidades de decisión; y c) espacio como un campo de objetos homogéneo (Lasuen, 1969, Darwent, 1969 y Moseley, 1974).

${ }^{2}$ Para Perroux, como se advierte, el término polarización refiere a la intensidad de las transacciones interfirmas o interindustrias, lo cual refleja la conformación de un polo de crecimiento (Moseley, 1974 y Parr, 1999a). 
un polo de crecimiento es "una unidad propulsora en relación con el ambiente circundante" (Perroux, citado en Moyoshi, 1997: 5).

Para los setenta, Richardson formula de manera conjunta los elementos básicos de una teoría dinámica del desarrollo espacial y de una teoría del desarrollo económico regional, advirtiendo que "la estructura espacial de una región y el tamaño y dispersión de sus ciudades quizá sean factores cruciales en la explicación del crecimiento regional potencial" (Richardson, 1973a, citado en Moseley, 1974: 7).

Richardson habla de "puntos de crecimiento" en la estructura naturalmente evolucionada de una región nodal. Una región nodal se conforma por una jerarquía de asentamientos funcionalmente interrelacionados. Estas interrelaciones funcionales revelan flujos que no se producen al mismo tiempo en el espacio, ${ }^{3}$ que tienden a polarizarse hacia y desde un nodo dominante, normalmente a las grandes ciudades (Richardson, 1975).

En cada nodo hay una zona de influencia donde hay una interacción de muchos géneros y en la cual los flujos declinan a medida que nos alejamos del centro o "punto de crecimiento". En este sentido, la idea de los puntos de crecimiento es que "la actividad económica de una región tiende a aglomerarse en un pequeño número de puntos o nodos dominantes y que los flujos de polarización gravitarán hacia estos puntos en la región, pero la densidad de los flujos vendrá reducida por la distancia" (Richardson, 1975: 111).

Estos niveles de concentración se logran mediante la dirección oficial o por las economías de aglomeración ${ }^{4}$ que representan economías internas y externas para la empresa, sujetas a la concentración espacial de la actividad económica. Para Richardson (1975), las economías de escala externas proveen las principales ventajas de

\footnotetext{
${ }^{3}$ Las interrelaciones o vinculaciones funcionales en las regiones pueden referirse a muchas fuentes: canales de distribución de mercancías, los patrones de desplazamientos y las corrientes migratorias, densidades telefónicas y de otras comunicaciones, las áreas de captación de mano de obra y los patrones de desplazamiento diario al trabajo, por ejemplo.

4 Para una exploración detallada de este concepto, véase Moseley (1974), Richardson (1975) y Parr (1999a).
} 
aglomeración por lo que conviene tener presente las economías de urbanización, ${ }^{5}$ ya que la aglomeración espacial puede propiciar condiciones para el crecimiento económico y por la propensión de las ciudades a inculcar valores y actitudes favorables al crecimiento.

En los noventa, la corriente de autores que se inscriben en la nueva geografía económica (NGE) plantea que "la cuestión que define a la geografía económica es la necesidad de explicar la concentración de la población y de la actividad económica; a saber, la distinción entre cinturones industriales y agrícolas, la existencia de las ciudades y el papel de los núcleos industriales" (Fujita, et al., 1999: 14). En este sentido, la NGE se enfoca en los elementos y las categorías que puedan explicar el surgimiento, desarrollo y declive de las estructuras regionales y urbanas.

En el contexto regional, construyen un modelo para una economía monopolísticamente competitiva y muestran cómo la interacción entre rendimientos crecientes a nivel de empresa, los costos de transporte de los productos manufacturados y el factor movilidad dan lugar a la aparición de economías de aglomeración y a la modificación posterior de la estructura económica espacial. En particular, se muestra cómo en una economía bi-regional se pueden diferenciar un núcleo industrializado y una periferia agrícola; esto es, se identifica el surgimiento de un patrón de desarrollo regional centro-periferia.

En el patrón centro-periferia, las vinculaciones verticales se sustentan en dos aspectos: a) la renta es mayor en la localización industrializada porque dispone de toda la renta generada en dicho sector; $\mathrm{y} b$ ) los precios son mayores en la localidad agrícola que debe importar todos los productos manufacturados. Así, cuando el sector industrial es grande, el potencial de economías de escala y el bajo costo de transporte favorecen la aglomeración de la producción y la

\footnotetext{
${ }^{5}$ Son economías externas a la empresa y la industria, surgen cuando la actividad económica involucra empresas pertenecientes a diferentes industrias: incluye el acceso a un mercado más grande, disminución de costos por la disponibilidad compartida de servicios públicos, un amplio rango de servicios especializados (comerciales, de banca y financieros), así como la existencia de instalaciones sociales, culturales y de ocio que influyen en las decisiones de localización. Véase Moseley (1974), Richardson (1975) y Parr (1999a).
} 
industria genera importantes vinculaciones progresivas a través de la oferta y regresivas a través de la demanda (Richardson, 1975).

También se construye un modelo urbano, cuya estructura guarda estrecha relación con el modelo centro-periferia. La economía tiene un sector agrícola y otro industrial, el primero produce un único bien homogéneo y el segundo bienes diferenciados. Los trabajadores son libres para ocuparse en uno $\mathrm{u}$ otro sector productivo, y también se incluye un factor inmóvil que crea fuerzas centrífugas: el suelo. El modelo genera fuerzas centrípetas y centrífugas, como en el modelo regional, que surgen con la interacción entre las economías de escala, los costos de transporte y la movilidad de los factores productivos.

Entonces, si los productos manufacturados están lo suficientemente diferenciados entre sí y la población no es excesivamente grande, las fuerzas centrípetas superan a las centrífugas permitiendo la aglomeración de la fabricación de productos manufacturados en una única ciudad, por lo que la geografía de la economía puede ser monocéntrica. Pero si la producción manufacturera no está muy diferenciada y la población es suficientemente grande, el uso agrícola de la tierra en la economía monocéntrica se extenderá lejos de la ciudad central y los productores individuales tendrán incentivos para ubicarse lejos de la ciudad, por lo que tenderán a emerger nuevas ciudades. Así se transita de una geografía monocéntrica a la configuración espacial de un sistema de ciudades.

\section{Estudios empíricos sobre el tema}

Pocos estudios atienden las diferencias socioeconómicas interregionales. Lipshitz et al. (1998) aplicaron la técnica Co-Plot en Israel; las observaciones comprendieron 81 ciudades y utilizaron cinco variables socioeconómicas; el método proporciona la clasificación de las 81 ciudades según nivel socioeconómico y un mapa socioeconómivo espacial, esto es, la distribución geográfica de las ciudades según nivel de desarrollo socioeconómico, lo que da cuenta de la polarización del desarrollo en Israel a comienzos de los noventa. 
En México, el estudio del crecimiento y el desarrollo regional ha ofrecido más atención al acercamiento o distanciamiento de variables como el PIB per cápita, el РІв por entidades o el empleo. En cuanto al PIB por entidades, se encontró una leve reducción en las disparidades durante 1970-1996 con alta concentración en el centro del país (Garza, 2000); sin embargo, hay quienes advierten nuevos centros industriales, los cuales han inducido un cambio en la distribución espacial de las actividades económicas en las últimas décadas (Olivera, 2001 y Corona, 2003). Esto se atribuye a la inserción de México en la división internacional del trabajo, la apertura económica y un menor dinamismo del mercado interno.

Hay algunos trabajos que tratan el desarrollo regional en las entidades y en la perspectiva de las disparidades socioeconómicas. Para Jalisco, Arroyo (1993), aplicando el método de análisis factorial de componentes principales a 11 variables socioeconómicas de 1970 y 1990, encontró que no aumenta la polarización del desarrollo regional durante 1970-1990. Guadalajara y Zapopan se mantuvieron en nivel alto de desarrollo y Tlaquepaque pasó a formar parte de dicha categoría en el periodo de análisis. Un municipio cabeza de región, Tamazula de Gordiano, pasó del estrato bajo en 1970 a nivel medio en 1990; otros tres municipios que encabezan regiones, La Barca, Lagos de Moreno y Tepatitlán de Morelos, se mantuvieron en nivel medio. El resto de las cabeceras regionales siguieron en el estrato alto, aunque tuvieron disminuciones en su nivel, con excepción de Puerto Vallarta.

Para Michoacán, Navarro et al. (2000) utilizaron una propuesta metodológica que llaman Índice de Desarrollo Socioeconómico Municipal con Distribución del Ingreso (IDSEM ${ }^{\mathrm{DIST}}$ ), ya que incorporan indicadores distributivos entre los diez que comprende dicha metodología. Para el 2000, los municipios que registraron más alto desarrollo económico y social fueron Jacona, Morelia, Zamora, Azuayo, La Piedad, Lázaro Cárdenas, Uruapan y Zacapu; esto es, ocho de 113 municipios marcaban la pauta de las condiciones sociales y económicas; además, éstos son municipios en los que se encuentran las ciudades de mayor importancia en Michoacán. 


\section{Especificación del modelo estadístico y econométrico}

Se estima un Índice de Desarrollo Socioeconómico Municipal (IDSEM) para cada municipio de Sonora, mediante la técnica estadística del análisis factorial de componentes principales. ${ }^{6}$ Los IDSEM que el método proporciona se calcularon con base en 10 indicadores obtenidos a partir de la información proporcionada en el Sistema de Información Municipal de Bases de Datos (SIMBAD). Se considera $I_{i j}$ como el indicador socioeconómico $j$ para cada municipio $i$ de la entidad, donde $j=$ $1,2, \ldots, 10$; e $i=1,2, \ldots, 70$. Los indicadores son los siguientes:

$I_{i 1}$ Porcentaje de población económicamente activa (PEA) ocupada cuyo ingreso es de dos salarios mínimos y más, ${ }^{7}$

$I_{i 2}$ Porcentaje de PEA ocupada en la industria y en servicios,

$I_{i 3}$ Porcentaje de población urbana, ${ }^{8}$

$I_{i 4}$ Porcentaje de población de 15 años o más alfabeta,

$I_{i 5}$ Porcentaje de población de 15 años o más con algún grado terminado de educación preparatoria,

$I_{i 6}$ Porcentaje de población de 18 años o más con algún grado terminado de educación profesional,

$I_{i 7}$ Porcentaje de población en viviendas con agua entubada,

$I_{i 8}$ Porcentaje de población en viviendas con drenaje;

$I_{i 9}$ Porcentaje de población en viviendas con electricidad; y,

$I_{i 10}$ Porcentaje de población en viviendas sin piso de tierra.

Una vez calculados los indicadores socioeconómicos, es necesario construir una medida resumen que dé cuenta del desarrollo. De esta

\footnotetext{
${ }^{6}$ Para una explicación detallada de esta técnica véase Vidal Díaz de Rada (2001), capítulos 1, 2 y 3. También María Álvarez (2000).

${ }^{7}$ Un estudio de Conapo (1993) consideraba dos salarios mínimos en 1990 como el ingreso requerido por un trabajador para satisfacer sus necesidades mínimas; por tanto, en este trabajo se asume que la PEA con dos salarios mínimos y más está en posición de satisfacer más allá de sus necesidades mínimas.

${ }^{8}$ No se adopta el criterio de INEGI, que considera como localidades urbanas aquellas que albergan 2500 habitantes y más. El criterio seleccionado define como localidades urbanas aquellas de 15 mil habitantes y más y tiene por objetivo la identificación de las ciudades, con ello la captación de los municipios verdaderamente urbanizados. Al respecto, véase Navarro (2002).
} 
manera, se recurre al análisis factorial de componentes principales, el cual es un método estadístico que transforma un conjunto de variables o indicadores en uno nuevo, lo que ofrece una interpretación más sencilla del fenómeno en estudio. Para ello se requiere que las variables originales presenten cierto nivel de correlación, lo que es evidente en el caso de los indicadores utilizados (véase apéndice técnico, cuadro A).

Antes de aplicar el método citado para el cálculo del IDSEM, a fin de eliminar los efectos de escala entre las variables o los efectos de varianzas notablemente distantes, éstas se estandarizan mediante el promedio aritmético y la desviación estándar en cada uno de los municipios de la siguiente forma:

$$
z_{i j}=I_{i j}-I_{j} / d s_{j}
$$

Donde:

$z_{i j}$ es el indicador estandarizado $j(j=1, \ldots, 10)$ del municipio $i$ $(i=1, \ldots, 70)$,

$I_{i j}$ es el indicador socioeconómico $j$, de la unidad de análisis $i$,

$I_{j}$ es el promedio aritmético de los valores del indicador $j, y$

$d s_{j}$ es la desviación estándar insesgada del indicador socioeconómico $j$.

Estas nuevas variables tienen como característica un promedio aritmético o media igual a cero, mientras que la varianza y la desviación estándar son iguales a uno. En la estimación del IDSEM se utilizó el paquete sPss versión 10 que proporciona componentes principales estandarizados, con media cero y desviación estándar uno. De tal manera, los IDSEM corresponden a la primera componente estandarizada de cada municipio, que es una combinación lineal de las 10 variables estandarizadas, esto es:

$$
Y_{i 1}=\text { IDSEMi }=\sum_{j=1}^{10} c_{1} z_{i j}=c_{1} z_{i 1}+c_{1} z_{i 2}+\ldots+c_{1} z_{i 10}
$$

Donde:

$Y_{i 1} \quad$ es el valor del municipio $i$ en la primera componente principal estandarizada. 
Cuadro 1. Estratos para la clasificación del Índice de Desarrollo Socioeconómico Municipal (LDSEM), 2000

\begin{tabular}{lc}
\hline Año 2000 & IDSEM \\
\hline$[2.056050,0.419782]$ & Alto \\
{$[0.419781,-0.446091]$} & Medio \\
{$[-0.446090,-2.8253101$} & Bajo \\
\hline
\end{tabular}

Fuente: Estimación propia con base en INEGI-Sistema de Información Municipal de Bases de Datos (Simbad) y en el Método Estadístico de Componentes Principales, 2000.

$I_{D S E M}$ es el valor del Índice de Desarrollo Socioeconómico en el municipio $i$,

$c_{j} \quad$ es el ponderador del indicador $j$ para determinar la primera componente principal estandarizada, $\mathrm{y}$

$z_{i j} \quad$ es el indicador estandarizado $j$ del municipio $i$.

El Índice de Desarrollo Socioeconómico asume valores positivos o negativos. Los valores positivos mayores indican un alto desarrollo socioeconómico municipal, mientras que entre más elevado sea el valor negativo más bajo será el desarrollo socioeconómico en los municipios. Para el 2000, los IDSEM obtenidos varían desde un valor máximo de 2.0560 hasta un valor de -2.8253 (cuadro 1 ).

\section{El modelo econométrico}

Para comprobar la hipótesis se utiliza un modelo de regresión lineal múltiple; las variables se formulan con base en la revisión teórica. La idea a explorar es que el desarrollo socioeconómico de la población municipal lo determinan las siguientes variables: el índice de urbanización municipal, las unidades económicas de la industria manufacturera en los municipios, la población económica activa ocupada en actividades agrícolas y el valor agregado de las manufacturas en cada municipio. El modelo econométrico quedó de la siguiente forma: 
$I_{D S E M}=\beta o+\beta_{1} I u r b i+\beta_{2} U E$ Eindi $+\beta_{3} P E A-A g i+\beta_{4} V_{A m a n}+\varepsilon_{l}$

Donde:

$I_{D S E M}=$ Índice de Desarrollo Socioeconómico Municipal.

Iurbi $=$ Índice de Urbanización, estimado de acuerdo al índice de Unikel.

UEind $i=$ Unidades productivas en la industria manufacturera del municipio $i$, medidas como porcentaje respecto al total de unidades productivas en el municipio.

$P E A-A g_{i}=$ Población económicamente activa ocupada en actividades agrícolas del municipio $i$, medida como porcentaje respecto a la PEA total en el municipio.

$V A m a n_{i}=$ Valor agregado de la industria manufacturera en el municipio $i$, medido como porcentaje respecto al valor agregado total en el municipio.

$\varepsilon_{i}=\quad$ Término de error.

Para la regresión del modelo se utilizaron datos proporcionados por el censo económico del año 1999, información que en realidad corresponde a 1998, que es el año cuando se realizó el levantamiento censal; no obstante, para efecto del presente trabajo se asume que no hay una gran variación en la información durante dos años, por lo que los datos de 1999 se utilizan para el año 2000. El modelo es de corte transversal y se aplican mínimos cuadrados ordinarios.

\section{Concentración económica y desarrollo regional en el estado de Sonora}

Antes de analizar el desarrollo regional, conviene establecer en forma somera la ubicación geográfica y una breve caracterización socioeconómica de Sonora. El estado se localiza al noroeste de México y es una de las seis entidades ${ }^{9}$ que colindan con Estados Unidos de

${ }^{9}$ Los cinco estados restantes son Baja California, Chihuahua, Coahuila, Nuevo León y Tamaulipas. 
América. También tiene colindancia terrestre con Baja California al oeste, con Chihuahua al este y con Sinaloa al sur. El estado de Baja California Sur tiene con Sonora límites marítimos (Montané, 1993).

La entidad cuenta con 70 municipios, su extensión territorial de 184934 km² le coloca en el segundo lugar nacional después de Chihuahua. ${ }^{10}$ La economía se conforma por sectores modernos como el agrícola y el industrial orientados a las exportaciones, principalmente la maquiladora. En el 2000 Sonora era el estado menos poblado $-2.27 \%$ respecto a la población total de México- y también la entidad que menos riqueza generaba en la frontera norte del país: un PIB estatal de 154658 millones de pesos que representa el 2.85\% del Рів nacional (Camberos, et al., 2003: 160, cuadro 1). Esto explica por qué Sonora, que genera menos riqueza, tiene un PIB per cápita mayor que Baja California y Tamaulipas.

En este año, Sonora también genera la menor cantidad de empleos (Camberos, ibid). El estado tenía un promedio escolar de 8.1 grados, superando el promedio de 7.5 nacional; el porcentaje de población analfabeta de 15 años y más era de $4.40 \%$ en el estado y de $9.46 \%$ el nacional; mientras que la esperanza de vida al nacer era 76.64 años en la entidad y a escala nacional de 75.95 años (Gobierno del Estado, 2000). El estado también destaca en este año con un porcentaje menor al 5\% en los siguientes indicadores: ocupantes en viviendas sin electricidad, en viviendas sin agua entubada y sin drenaje ni excusado; además, estos porcentajes son menores a los del país (Camberos, ibid.).

Estos datos confirman la percepción de una "prosperidad inherente" a los Estados de la frontera norte de México; sin embargo, las cifras del PIB muestran que Sonora no es precisamente la entidad más próspera. Además, es obvio que las cifras agregadas del desarrollo económico no reflejan las disparidades regionales, ni el hecho de que el crecimiento no se comparte igualmente por todos los grupos sociales y al interior de cada uno de los Estados (OECD, 2003). En este sentido, cobra relevancia el estudio y análisis de la distribución espacial del desarrollo socioeconómico en Sonora. 
En diferentes estudios tradicionalmente se reconocen tres grandes regiones en el estado de Sonora, la costa, la frontera y la sierra ${ }^{11}$ (Gracida, 2002; Ruiz, 1999; Wong, 1994); para facilitar el análisis se consideran estas tres regiones en el presente trabajo (mapa 1). La estimación del IDSEM ayuda a identificar las inequidades existentes en el espacio económico regional de Sonora, como se verá a continuación.

\section{Panorama del desarrollo socioeconómico regional en Sonora para el año 2000}

En el 2000 había 22 municipios con alto desarrollo socioeconómico, en los cuales habitaban 1885839 personas, el 85.06\% de la población total en Sonora (cuadro 2). Destacan Cajeme, Cananea, Hermosillo y Nogales, que tenían el IDSEM positivo más alto en este estrato de desarrollo, y les seguían los municipios de Agua Prieta, Fronteras, Magdalena, Moctezuma, Naco, Nacozari de García, Puerto Peñasco y San Luis Río Colorado. Estaban relativamente en peor situación, con el IDSEM positivo más bajo, Benjamín Hill, Caborca, Cumpas, Empalme, Granados, Guaymas, Huépac, Mazatán, Navojoa y Santa Ana.

En cuanto a proporción de población, sobresalen dos municipios donde están los principales centros urbanos del estado: Hermosillo y Cajeme $^{12}$ (Mendez, 2002), éstos concentraban 966119 habitantes que

${ }^{11}$ Los municipios que comprende cada región se definen "ajustando" las 12 regiones geoeconómicas de Sonora, de acuerdo al Comité para la Planeación del Desarrollo (Coplades): La Costa: Altar, Atil, Bácum, Caborca, Cajeme, Etchojoa, Hermosillo, Huatabampo, Navojoa, Oquitoa, Pitiquito, Plutarco Elías Calles, San Luis Río Colorado, Saric y Tubutama; La Frontera: Agua Prieta, Bacoachi, Benjamín Hill, Cananea, Cucurpe, Fronteras, Imuris, Magdalena, Naco, Nacozari de García, Nogales, Santa Ana, Santa Cruz y Trincheras; y La Sierra: Álamos, Aconchi, Arivechi, Arizpe, Bacadéhuachi, Bacanora, Bacerac, Banámichi, Baviácora, Bavispe, Carbó, Cumpas, Divisaderos, Granados, Huachineras, Huásabas Huépac, La Colorada, Mazatán, Moctezuma, Nácori Chico, Onavas, Opodepe, Quiriego, Rayón, Rosario Tesopaco, Sahuaripa, San Felipe, San Javier, San Miguel de Horcaditas, San Pedro de la Cueva, Soyopa, Suaqui Grande, Tepache, Ures, Villa Hidalgo, Villa Pesqueira y Yécora.

12 Respectivamente, en estos municipios se localizan Hermosillo -capital del estado- y Ciudad Obregón. 


\section{Mapa 1}

Regiones de Sonora

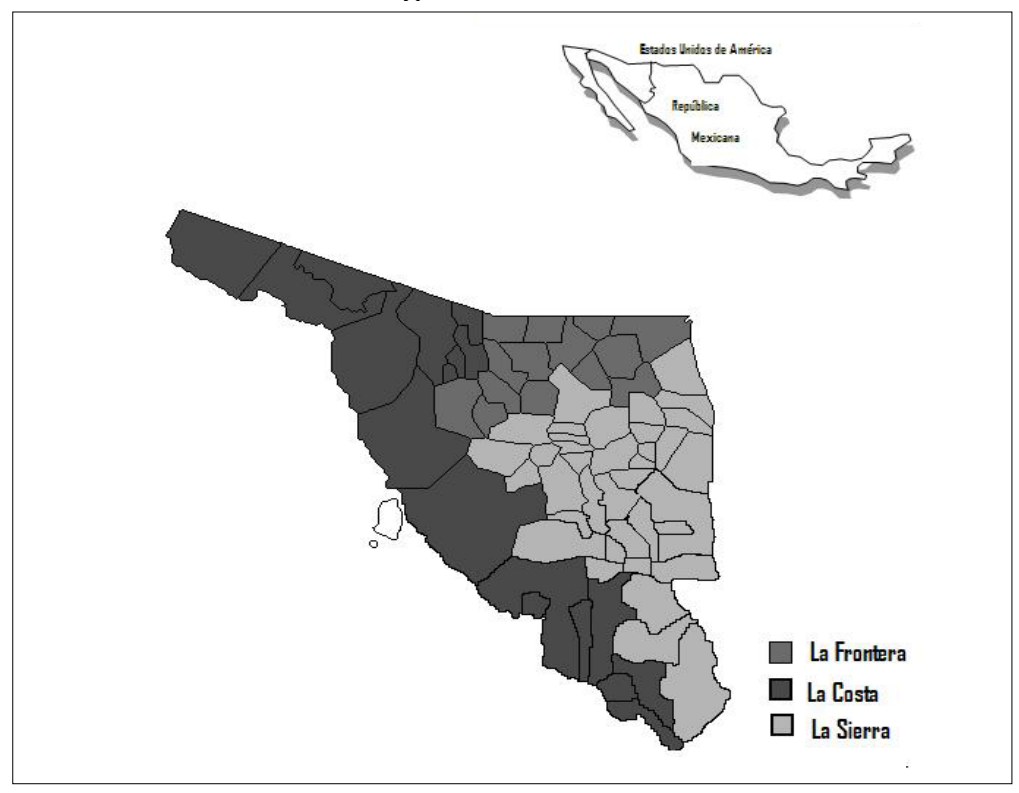

Fuente: Elaboración propia con base en Coplades, Gobierno del Estado de Sonora.

equivalían al $43.58 \%$ de la población total en el estado. Una proporción acumulada menor se observa en Nogales (7.21\%), San Luis Río Colorado (6.54\%), Guaymas (6.50\%), Navojoa (6.34\%), Caborca (3.14\%), Agua Prieta (2.79\%), Empalme (2.25), Cananea (1.45), Puerto Peñasco (1.41\%) y Magdalena (1.10\%), que sumaban 858576 habitantes, equivalentes al $38.72 \%$ de la población estatal. Los 10 municipios restantes con alto desarrollo socioeconómico sumaban 61144 habitantes, apenas 2.75\% respecto a la población total en el estado.

Esto implica que en el 2000, sólo 12 de los 70 municipios que constituían la entidad concentraban a 1824695 habitantes, los que significaban $82.30 \%$ de la población total en Sonora. Es importante señalar que sólo en Hermosillo y Cajeme residía el 43.58\% de dicha población, más de la mitad de la población residente en los 22 municipios clasificados con un desarrollo socioeconómico alto y más de la tercera parte respecto a la población total en el estado. 
Cuadro 2. Municipios con alto nivel de desarrollo socioeconómico en 2000

\begin{tabular}{llrrr}
\hline & Municipio & IDSEM & Población & $\%$ \\
\hline 002 & Agua Prieta & 1.41170 & 61,944 & 2.79 \\
016 & Benjamín Hil & 0.77696 & 5,732 & 0.26 \\
017 & Caborca & 0.53350 & 69,516 & 3.14 \\
018 & Cajeme & 1.60635 & 356,290 & 16.07 \\
019 & Cananea & 2.05605 & 32,061 & 1.45 \\
023 & Cumpas & 0.65024 & 6,202 & 0.28 \\
025 & Empalme & 0.72357 & 49,987 & 2.25 \\
027 & Fronteras & 1.02011 & 7,801 & 0.35 \\
028 & Granados & 0.68971 & 1,235 & 0.06 \\
029 & Guaymas & 0.88091 & 144,021 & 6.50 \\
030 & Hermosillo & 1.95858 & 609,829 & 27.51 \\
034 & Huépac & 0.52476 & 1,142 & 0.05 \\
036 & Magdalena & 1.37325 & 24,447 & 1.10 \\
037 & Mazatán & 0.52279 & 1,584 & 0.07 \\
038 & Moctezuma & 1.21830 & 4,187 & 0.19 \\
039 & Naco & 1.01874 & 5,370 & 0.24 \\
041 & Nacozari de García & 1.25825 & 14,365 & 0.65 \\
042 & Navojoa & 0.44613 & 140,650 & 6.34 \\
043 & Nogales & 1.48652 & 159,787 & 7.21 \\
048 & Puerto Peñasco & 1.38050 & 31,157 & 1.41 \\
055 & San Luis & & & \\
& Río Colorado & 1.18993 & 145,006 & 6.54 \\
058 & Santa Ana & 0.94221 & 13,526 & 0.61 \\
\hline
\end{tabular}

Fuente: Estimación propia con base en INEGI-Simbad 2000 y en el Método Estadístico de Componentes Principales.

Nota: El porcentaje es respecto a la población total del estado.

En el estrato de desarrollo socioeconómico medio había 25 municipios y en ellos vivían 87559 personas, apenas 3.95\% de la población Sonorense (cuadro 3). En este estrato, 14 municipios tenían relativamente la mejor situación: Aconchi, Atil, ${ }^{13}$ Banámichi, Baviácora,

13 A los municipios de Altar, Atil, Oquitoa, Pitiquito, Sáric y Tubutama, a mitad de los noventa se les conocía como "pueblos del tercer mundo" en los círculos gubernamentales, pues estaban "relegados" de los programas gubernamentales (Wong, 1994). No obstante, las estimaciones del IDSEM muestran que no hay tal "tercer mundo" ya que sólo Tubutama tenía un IDSEM bajo en el 2000 . 
Cuadro 3. Municipios con nivel medio de desarrollo socioeconómico en 2000

\begin{tabular}{|c|c|c|c|c|}
\hline & Municipio & IDSEM & Población & $\%$ \\
\hline 001 & Aconchi & 0.27684 & 2,420 & 0.11 \\
\hline 004 & Altar & -0.16316 & 7,253 & 0.33 \\
\hline 005 & Arivechi & -0.27098 & 1,484 & 0.07 \\
\hline 006 & Arizpe & -0.38775 & 3,396 & 0.15 \\
\hline 007 & Atil & 0.41905 & 718 & 0.03 \\
\hline 008 & Bacadéhuachi & -0.16334 & 1,348 & 0.06 \\
\hline 011 & Bacoachi & -0.29285 & 1,496 & 0.07 \\
\hline 013 & Banámichi & 0.26676 & 1,484 & 0.07 \\
\hline 014 & Baviácora & 0.24074 & 3,724 & 0.17 \\
\hline 020 & Carbó & -0.22204 & 4,984 & 0.22 \\
\hline 024 & Divisaderos & 0.25616 & 825 & 0.04 \\
\hline 032 & Huásabas & 0.16959 & 966 & 0.04 \\
\hline 035 & Imuris & 0.33922 & 9,988 & 0.45 \\
\hline 046 & 0quitoa & -0.04316 & 402 & 0.02 \\
\hline 047 & Pitiquito & 0.32161 & 9,236 & 0.42 \\
\hline 050 & Rayón & -0.28414 & 1,591 & 0.07 \\
\hline 052 & Sahuaripa & -0.30676 & 6,400 & 0.29 \\
\hline \multirow[t]{2}{*}{053} & San Felipe & & & \\
\hline & de Jesús & 0.42125 & 416 & 0.02 \\
\hline 059 & Santa Cruz & -0.23246 & 1,628 & 0.07 \\
\hline 060 & Sáric & -0.43929 & 2,257 & 0.10 \\
\hline 062 & Suaqui Grande & 0.32728 & 1,175 & 0.05 \\
\hline 063 & Tepache & 0.40104 & 1,539 & 0.07 \\
\hline 066 & Ures & 0.38364 & 9,565 & 0.43 \\
\hline 067 & Villa Hidalgo & 0.16172 & 1,986 & 0.09 \\
\hline \multirow[t]{2}{*}{070} & General Plutarco & & & \\
\hline & Elías Calles & 0.36612 & 11,278 & 0.51 \\
\hline
\end{tabular}

Fuente: Estimación propia con base en INEGI-Simbad 2000 y en el Método Estadístico de Componentes Principales.

Nota: El porcentaje es respecto a la población total del estado.

Divisaderos, Huásabas, Imuris, Pitiquito, San Felipe de Jesús, Suaqui Grande, Tepache, Ures, Villa Hidalgo y Plutarco Elías Calles. En situación intermedia estaba sólo el municipio de Oquitoa, seguido por 10 municipios en "peor" situación relativamente con un IDSEM negativo: Altar, Arivechi, Arizpe, Bacadéhuachi, Bacoachi, Carbó, Rayón, Sahuaripa, Santa Cruz y Sáric. 
La distribución de la población en el estrato de desarrollo medio muestra una gran dispersión de la misma. Los municipios más poblados eran General Plutarco Elías Calles, Pitiquito y otros dos tipificados como nuevos centros maquileros, Imuris y Ures, que en conjunto sumaban 40067 habitantes que equivalían al 1.80\% de la población total en Sonora, casi la mitad de la población en este estrato de desarrollo. En los 21 municipios restantes vivían apenas 47492 personas, el $2.14 \%$ de los habitantes en el estado.

Había 23 municipios con desarrollo socioeconómico bajo, habitados por 243571 personas que equivalían al 10.99\% de la población total en el estado (cuadro 4). Entre éstos, 14 municipios tenían un IDSEM cuyo valor negativo era el más bajo, por lo que relativamente estaban en "mejor" situación: Bacanora, Bacerac, Bácum, Bavispe, La Colorada, Huachinera, Onavas, Opodepe, San Javier, San Pedro de la Cueva, Soyopa, Trincheras, Tubutama y un municipio netamente urbano: Huatabampo. En situación "intermedia" estaban Cucurpe, Etchojoa y Nácori Chico. En la peor situación estaban seis municipios: Álamos, Quiriego, Rosario, San Miguel de Horcasitas, Villa Pesqueira y Yécora.

En cuanto a la distribución de la población sobresalen los municipios de Etchojoa y Huatabampo, que sumaban 154238 habitantes, equivalentes al $6.96 \%$ de la población total en el estado. A la vez, estos dos municipios comprenden el $63.32 \%$ de la población residente en los 23 municipios clasificados con nivel de desarrollo socioeconómico bajo. Ambos municipios están enclavados en una zona agrícola por excelencia: el Valle del Mayo.

Una proporción de población acumulada menor se observa en los municipios de Álamos (1.13\%), Bácum (0.96\%), Yécora $(0.27 \%)$, San Miguel de Horcasitas (0.25\%), Rosario (0.25\%) y Quiriego $(0.15 \%)$, los que sumaban 66936 habitantes y equivalían al 3.01\% de la población total en el estado. Esto significa que el $90.80 \%$ de la población cuyo nivel de desarrollo socioeconómico era bajo habitaba sólo en ocho de los 23 municipios en este estrato de desarrollo. Los 15 municipios restantes estaban habitados por 22397 personas, apenas el $1.01 \%$ de la población total en el estado.

El mapa 2 provee una visión más clara de la distribución espacial del desarrollo socioeconómico. En La Costa se observan ocho munici- 
Cuadro 4. Municipios que tenían Bajo nivel de desarrollo socioeconómico en el 2000

\begin{tabular}{|c|c|c|c|c|}
\hline & Municipio & IDSEM & Población & $\%$ \\
\hline 003 & Álamos & -2.34419 & 25,152 & 1.13 \\
\hline 009 & Bacanora & -0.56407 & 943 & 0.04 \\
\hline 010 & Bacerac & -0.91551 & 1,366 & 0.06 \\
\hline 012 & Bácum & -0.82016 & 21,322 & 0.96 \\
\hline 015 & Bavispe & -0.75268 & 1,377 & 0.06 \\
\hline 021 & Colorada, La & -0.66190 & 2,306 & 0.10 \\
\hline 022 & Cucurpe & -1.47482 & 937 & 0.04 \\
\hline 026 & Etchojoa & -1.07104 & 77,942 & 3.52 \\
\hline 031 & Huachinera & -0.66028 & 1,147 & 0.05 \\
\hline 033 & Huatabampo & -0.55812 & 76,296 & 3.44 \\
\hline 040 & Nácori Chico & -1.11003 & 2,236 & 0.10 \\
\hline 044 & Onavas & -0.94171 & 479 & 0.02 \\
\hline 045 & Opodepe & -0.90331 & 2,831 & 0.13 \\
\hline 049 & Quiriego & -2.82531 & 3,335 & 0.15 \\
\hline 051 & Rosario & -1.86510 & 5,432 & 0.25 \\
\hline 054 & San Javier & -0.49279 & 279 & 0.01 \\
\hline \multirow[t]{2}{*}{056} & San Miguel & & & \\
\hline & de Horcasitas & -1.86201 & 5,626 & 0.25 \\
\hline \multirow[t]{2}{*}{057} & San Pedro & & & \\
\hline & de la Cueva & -0.66770 & 1,703 & 0.08 \\
\hline 061 & Soyopa & -0.45970 & 1,649 & 0.07 \\
\hline 064 & Trincheras & -0.96618 & 1,756 & 0.08 \\
\hline 065 & Tubutama & -0.91258 & 1,798 & 0.08 \\
\hline 068 & Villa Pesqueira & -0.47699 & 1,590 & 0.07 \\
\hline 069 & Yécora & -1.90790 & 6,069 & 0.27 \\
\hline
\end{tabular}

Fuente: Estimación propia con base en INEGI-Simbad 2000 y en el Método Estadístico de Componentes Principales.

Nota: El porcentaje es respecto a la población total del estado.

pios clasificados en el estrato de desarrollo socioeconómico alto, los cuales se identifican como municipios netamente urbanos y donde se ubican ciudades como Hermosillo, Obregón, Guaymas, Empalme, Navojoa, Caborca, Puerto Peñasco y San Luis Río Colorado. Estos municipios tienen economías que están relativamente diversificadas, principalmente Hermosillo, donde está la ciudad capital del estado. 


\section{Mapa 2}

Sonora. Municipios según nivel de desarrollo socioeconómico, 2000

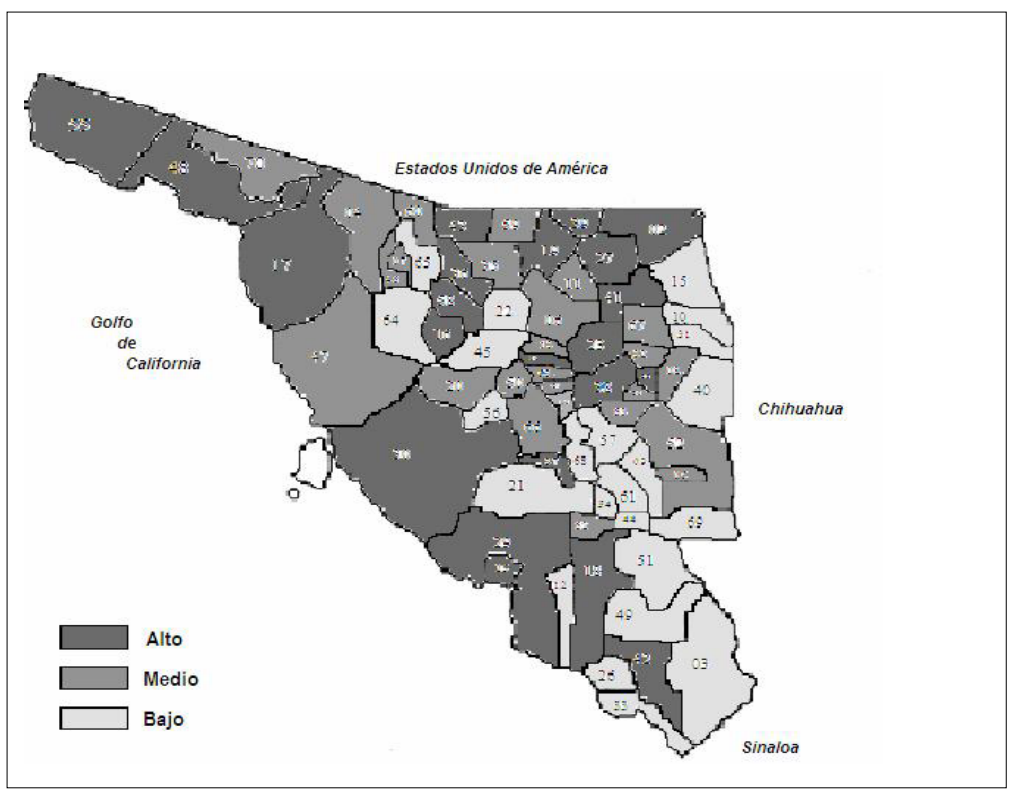

Fuente: Elaboración propia con base en INEGI y en el Método Estadístico de Componentes Principales.

En la frontera sobresalen los municipios de Nogales y Agua Prieta, que son municipios urbanizados donde sentó sus reales la actividad maquiladora desde los setenta, así como el comercio, los servicios aduaneros y el transporte. Próximos a Nogales están los municipios de Magdalena, Benjamín Hill y Santa Ana, que además de tener una base económica agropecuaria se insertan en el corredor maquilero Nogales-Guaymas-Empalme, donde tienen lugar las operaciones de mayor antigüedad (Wong, 1993).

En la frontera también aparecen con nivel de desarrollo alto los municipios de Cananea y Nacozari, los cuales se caracterizan por la preeminencia de la actividad minera. En la parte norte de la sierra había cuatro municipios cuyo nivel de desarrollo socioeconómico era alto en el 2000: Cumpas, Granados, Huépac y otro de los que se han 
identificado como nuevos centros maquileros: Moctezuma; aunque debe señalarse que de manera tradicional los municipios de la sierra han sustentado su economía principalmente en las actividades agropecuarias de baja escala y en la pequeña minería.

En el estrato de desarrollo medio, el mismo mapa muestra la presencia de los municipios aludidos como el "tercer mundo" de Sonora: Altar, Atil, Oquitoa, Sáric y Pitiquito. Esto deja en claro que dichos municipios no están entre los más atrasados de la entidad, pese a que las actividades productivas principales en ellos son la agricultura y la ganadería en baja escala. En la frontera tres municipios tenían un desarrollo socioeconómico medio: Bacoachi, Imuris y Santa Cruz.

En la sierra había 17 municipios que tenían un desarrollo socioeconómico medio: 12 en la parte norte y cinco en la parte centro, entre ellos dos de los que han dado cabida al desarrollo maquilador en esta región: Moctezuma y Ures. Parecería que los municipios con nivel medio de desarrollo se benefician por la "vecindad" con los municipios que tienen alto desarrollo ya que frecuentemente son contiguos a éstos. Las principales actividades productivas son la ganadería y la agricultura en baja escala, así como la pequeña minería, y son municipios que cuentan con caminos carreteros que les comunican hacia la costa y hacia la frontera.

El mismo mapa 2 muestra cuatro municipios en a costa con desarrollo socioeconómico bajo: al norte Tubutama y al sur Bácum, Etchojoa y también un municipio urbano, Huatabampo. Es sorprendente que Huatabampo, siendo un municipio urbano, aparezca en este estrato de desarrollo, lo que pudiera ser producto de la crisis en las actividades del sector primario experimentada por la economía sonorense (Wong, 1994), aunada a la concentración del gasto público en Hermosillo y los municipios de la frontera (Rodríguez, 1998, citado en Camberos et al., 2001: 21).

En la frontera sólo Cucurpe y Trincheras tenían un desarrollo socioeconómico bajo. En la sierra había 17 municipios en este estrato de desarrollo: al norte estaban cinco de ellos: Bacerac, Bavispe, Huachinera, Nácori Chico y Opodepe; mientras que al centro había nueve: Bacanora, La Colorada, Onavas, San Javier, San Miguel de Horcasitas, San Pedro de la Cueva, Onavas, Soyopa, Villa Pesqueira 
y Yécora. En el sur de la sierra destacan en este estrato los municipios de Álamos, Quiriego y Rosario, cuya economía descansa en las actividades primarias.

Estos resultados son consistentes con los índices de pobreza regional para el 2000, donde los municipios al sur de la sierra (Alamos, Quiriego, Rosario), algunos de la sierra centro (Bacanora, Sahuaripa y Yécora), y otros al sur de la costa (Bácum, Etchojoa y Huatabampo en el Valle del Yaqui y Mayo- constituyen los municipios con más pobreza por capacidades y por patrimonio (Camberos et al., 2005). Pero no son consistentes si se compara el IDSEM obtenido en este estudio con el Índice de Desarrollo Humano (IDH) estimado por el Conapo, donde todos estos municipios aparecen con un IDH medio alto.

\section{Resultados en la estimación del modelo econométrico}

La regresión muestra un coeficiente de determinación de $62 \%$, lo que implica un alto poder explicativo del modelo (cuadro 5). El índice de urbanización y la PEA agrícola presentan el signo esperado y son significativos estadísticamente. También la probabilidad inferior al $5 \%$ del estadístico " $\mathrm{F}$ " muestra que estas variables en conjunto resultan significativas en términos estadísticos, indicando una relación expli-

Cuadro 5. Valores estadísticos en la estimación del modelo econométrico

\begin{tabular}{lrrrr}
\hline Variable & Coeficiente & Error estándar & t-estadístico & Prob. \\
\hline C & 1.16213 & 0.212714 & 5.463356 & 0.000 \\
lurb & 0.009292 & 0.003542 & 2.623653 & 0.011 \\
PEA-Ag & -0.080576 & 0.011224 & -7.17918 & 0.000 \\
& & & & 70 \\
No. observaciones & & & 0.60 \\
R(ajustada) & & & 54.07247 \\
F-statistic & & & 0.000 \\
Prob(F-statistic) & & & 1.95 \\
D-W & & & \\
\hline
\end{tabular}

Fuente: Estimaciones propias mediante corte transversal. 
cativa de estas dos variables respecto al desarrollo socioeconómico de la población en los municipios.

La hipótesis de trabajo de esta investigación, que relaciona el desarrollo socioeconómico municipal con la concentración de la industria en los centros urbanos, lo que a la postre induce la concentración geográfica del desarrollo y a la vez de la población en los municipios con mayor desarrollo en el estado, debe rechazarse, ya que los resultados que arroja el modelo econométrico no la apoyan, pues las variables relacionadas con la industria no resultan significativas en términos estadísticos.

Finalmente, debe señalarse que la evidencia demuestra que la concentración industrial no es causante propiamente del desarrollo socioeconómico en el estado, y que más bien ésta debe estar acompañada de otros indicadores que no se incluyen en este estudio y que difícilmente pueden medirse a nivel municipal -por ejemplo la industria por sectores o subsectores, la inversión extranjera directa y el comercio internacional, entre otros-. Otra lectura de estos resultados pudiera llevar a aceptar la lógica de los mismos, pues el estado de Sonora, más que una entidad industrializada históricamente, se ha caracterizado por la preponderancia de las actividades agropecuarias.

\section{Conclusiones}

La revisión de las principales teorías o enfoques sobre concentración económica y desarrollo regional ha permitido dilucidar la "naturaleza" desigual que se presenta en el proceso de desarrollo, lo cual lleva a concebir la conformación de polos, nodos o centros de desarrollo como una situación inherente en el proceso del crecimiento económico y el desarrollo regional.

Se ha podido constatar la concentración del desarrollo en la población de los municipios urbanizados en el estado y también en algunos municipios que les eran contiguos, lo que da lugar a un patrón de desarrollo socioeconómico alto -también se hicieron evidentes un patrón de desarrollo medio y uno bajo-. Este patrón de desarrollo 
socioeconómico alto es visible en los municipios de las tres regiones en la entidad, particularmente en La Costa y La Frontera. En La Sierra sobresale con desarrollo socioeconómico alto, la población de municipios que en los noventa dieron margen a la actividad maquiladora, como son Imuris, Ures y Moctezuma.

Había 12 municipios netamente urbanos que concentraban el desarrollo socioeconómico en el estrato alto, les habitaban 1824839 personas, equivalentes al $82.30 \%$ de la población total en el estado; no obstante, significaban el $96.76 \%$ de las personas que vivían en los 22 municipios con desarrollo socioeconómico alto. Estos 12 municipios eran: Hermosillo, Cajeme, Nogales, San Luis Río Colorado, Guaymas, Navojoa, Caborca, Agua Prieta, Empalme, Cananea, Puerto Peñasco y Magdalena. Los municipios de Hermosillo y Cajeme se distinguen por albergar a 966119 habitantes, el $43.58 \%$ de la población total en el estado.

El modelo econométrico llevó a identificar que el desarrollo socioeconómico de la población en los municipios se explica en forma conjunta por el índice de urbanización y por la PEA cuya ocupación está ligada a las actividades agrícolas; mientras que la industria manufacturera no constituye una variable determinante en la explicación del desarrollo socioeconómico de la población municipal.

En este sentido, se rechaza la hipótesis de investigación que se diseñó para el estudio, ya que los resultados que arroja el modelo no la apoyan, pues las variables relacionadas con la industria no resultaron significativas en términos estadísticos. Otra interpretación de estos resultados pudiera llevar a aceptar la lógica de los mismos, pues Sonora, más que una entidad industrializada, históricamente se ha caracterizado por la preeminencia de las actividades de tipo agropecuario.

\section{Bibliografía}

Arroyo, A.J. (1993), "El desarrollo regional de Jalisco", Carta Económica Regional, Universidad de Guadalajara, mayo-junio, pp. 3-12.

Camberos, C.M. et al. (2005), "Niveles de pobreza en Sonora en los albores del siglo XXI, Boletín de los Sistemas Nacionales Estadístico 
y de Información Geográfica, vol. 1, núm. 2, septiembre-diciembre, INEGI, pp. 4-20.

y Jaime Yáñez (2003), "La informalidad de los mercados laborales de Sonora y la frontera norte de México", Región y Sociedad, El Colegio de Sonora, vol. XV, núm. 27, mayo-agosto, pp. 153-178.

y Joaquín Bracamontes (2001), "Pobreza y desequilibrios regionales en Sonora", Carta Económica Regional, Universidad de Guadalajara, julio-septiembre, pp. 17-24.

Corona, M.A. (2003), “Efectos de la globalización en la distribución espacial de las actividades económicas", Comercio Exterior, vol. 53, núm. 1, enero.

Darwent, D. (1969), "Growth Poles and Growth Centers in Regional Planning: A Review", Environment and Planning, vol. 1, pp. 532.

Díaz de Rada, V. (2000), Técnicas de análisis multivariante para investigación social y comercial. Ejemplos prácticos utilizando el SPSS versión 11, Ra-Ma.

Fujita, M., Paul Krugman y Anthony Venables (1999), The Spatial Economy: Cities, Regions and International Trade, MIT Press, Cambridge, MA.

Garza, G. (2000), “Tendencias de las desigualdades urbanas y regionales en México, 1970-1996", Estudios Demográficos y Urbanos, vol. 15, núm. 3, septiembre-diciembre.

Gobierno del Estado (2002), Quinto Informe de Gobierno, octubre.

Gracida, R. Juan J. (2001), La llegada de la modernización a Sonora. Establecimiento del ferrocarril, 1880-1897, UNISON.

INEGI-Simbad (2000), XII Censo General de Población y Vivienda. (1999), Censo Económico.

Lasuen, J.R. (1969), “On Growth-Poles”, Urban Studies, vol. 6, núm. 2, enero, pp. 137-161.

Lipshitz, G. y A. Raveh (1998), "Socioeconomic Differences among Localities: A New Method of Multivariate Analysis", Regional Studies, vol. 32.8, julio, pp. 747-757.

Méndez, S. Eloy (2002), Arquitectura y ciudades fronterizas, Universidad de Sonora. 
Meyer, Stephen y Hecht Alfred (1996), “University Growth Poles in Canada: An Empirical Assessment (1)", Canadian Journal of Regional Science, vol. XIX, núm. 3.

Miyoshi, T. (1997), Successes and Failures Associated with Growth Pole Strategies, disertación presentada en la Universidad de Manchester para obtener el grado de maestría en la Facultad de Ciencias Económicas y Sociales, Departamento de Estudios Económicos.

Montané , J.C. (1993), Atlas de Sonora, Gobierno del Estado de Sonora, Instituto Sonorense de Cultura, Hermosillo, Sonora.

Moseley, M. J. (1974), Growth Centres in Spatial Planning, Pergamon Press, Oxford.

Navarro, J.C. et al. (2000), "El desarrollo socioeconómico en Michoacán, 2000", en V.A. Acevedo, Economía y desarrollo regional en México.

OECD (2003), Territorial Reviews: México, OECD, París.

Olivera, G. (2001), "Implicaciones económico-territoriales del auge exportador mexicano", Estudios Demográficos y Urbanos, vol. 16, núm. 2, mayo-agosto.

Parr, J. B. (1999a), "Growth-Pole Strategies in Regional Economic Planning: A Retrospective View. Origens and Advocacy", Parte I en Urban Studies, vol. 36, núm. 7, 1195-1215, enero.

Pena T. Bernardo et al. (1999), Cien ejercicios de econometría, Pirámide, Madrid.

Richardson, H.W. (1975), Elementos de economía regional, Alianza, Madrid.

Unikel, L. et al. (1978), El desarrollo urbano de México, El Colegio de México, México.

Wong, G.P. (1994), “La reestructuración secto-espacial en Sonora: una tipología regional", en R.M. Vázquez (coord.), Las regiones ante la globalidad, SINO-Gobierno del Estado de Sonora. (1993), "La nueva industrialización rural en Sonora: de la villa a la fábrica global", en R.M. Vázquez (coord.), Sonora hacia el 2000: tendencias y desafíos, SINO-Gobierno del Estado de Sonora. 


\section{Apéndice técnico}

\section{Cuadro A. Carga de las variables* sobre el factor que mide el grado de desarrollo socioeconómico de la población en los municipios de Sonora, 2000 **}

\% PEA ocupada con 2 S.M. y más

0.767

$\%$ PEA en industria y servicios

$\%$ PEA urbana

\% Población alfabeta de 15 años y más

$\%$ Población de 15 años y más con algún grado de preparatoria

\% Población de 18 años y más con algún grado de educación profesional

\% Población con agua entubada

$\%$ Población con drenaje

$\%$ Población con electricidad

$\%$ Población sin piso de tierra

$\%$ de la varianza que representa el factor

* $\quad$ Factor Loading.

* Se utiliza el análisis de componentes principales, que sintetiza la variación común de un conjunto de variables en un factor (Diaz de Rada, 2000, capítulos 1-3).

Nota: Las variables consideradas se utilizan como indicadores del grado de desarrollo de la población; como se aprecia, el IDSEM (factor 1) se halla asociado positivamente con los diez indicadores. Las "cargas factoriales" sobre el primer factor representan el $83 \%$ de la variación común de estas variables y se interpreta como Índice de Desarrollo Socioeconómico Municipal (IDSEM), al considerarlo sintetizador de la variación común de las variables observables que ex profeso se seleccionaron como indicadores para medir el desarrollo en los municipios.

Fuente: Estimación propia con base en el INEGI-Sistema de Información Municipal de Bases de Datos (Simbad), y en el Método Estadístico de Componentes Principales, 2000. 